\title{
Classifying Human Manipulation Behavior
}

\author{
Ian M. Bullock and Aaron M. Dollar \\ Department of Mechanical Engineering and Materials Science \\ Yale University \\ New Haven, CT 06511, USA \\ ian.bullock@yale.edu, aaron.dollar@yale.edu
}

\begin{abstract}
This paper presents a taxonomy for detailed classification of human and anthropomorphic manipulation behavior. This hand-centric, motion-centric taxonomy differentiates tasks based on criteria such as object contact, prehension, and the nature of object motion relative to a hand frame. A sub-classification of the most dexterous categories, within-hand manipulation, is also presented, based on the principal axis of object rotation or translation in the hand frame. Principles for categorizing complex, multi-faceted tasks are also presented, along with illustrative examples. We hope that the proposed taxonomy will both establish a standard language around human and anthropomorphic manipulation as well as enable improved understanding of the differences in hand use for a wide variety of behavior. Although designed for human and anthropomorphic hands, the taxonomy might easily be extended to a wide range of robot manipulators and end-effectors.
\end{abstract}

\section{INTRODUCTION}

Human manipulation skill is unparalleled in the animal kingdom and is believed to have coevolved with our superior cognitive abilities, with each playing a complimentary role in the development of the other [1]. Each of our hands has as many controllable degrees of freedom (twenty one) as both arms, wrists, and one leg combined. Furthermore, the majority of tasks involve effort from multiple fingers, with the resulting combination of motions that can be produced to manipulate an object is therefore large.

Due to this complexity, classifying the manipulation capabilities of humans into a succinct categorization of distinct types is challenging and might be done a number of different ways, depending on the interests and perspective of the individual researcher. As engineers and roboticists, we are primarily interested in understanding the mechanics of human manipulation in order to impart those skills to mechanical hands for prosthetics and robot assistants. The classification presented in this paper focuses on the movements of the hand (not the wrist or arm) primarily during prehensile contact with an object. A hand centric taxonomy helps emphasize differences in hand function between tasks that might not be obvious from classifying full upper-limb or object movements. For the purposes of this paper, we loosely define manipulation as an action involving the hands, and typically resulting in motion of an external object.

The taxonomy presented in this paper sub-classifies human manipulation behavior according to the nature of the contact with external objects and the object motion imparted by the hand. While the classification is specifically focused on human and anthropomorphic manipulation, the same taxonomy or a slightly modified version can easily be applied to all finger-based mechanical hands. In addition to the fields of robotics and prosthetics, we expect this work to be useful in fields related to rehabilitation, occupational therapy, medicine, and psychology.

We begin this paper with a discussion of related work, providing an overview of other efforts towards classifying various types of hand or object movements. We then present our taxonomy, including descriptions of the binary tree leading to the various distinct sub-categorizations. Lastly, we provide a more detailed classification of dexterous within hand manipulation tasks; these require the most dexterity and are the focus of a number of ongoing efforts in robotic manipulation.

\section{RELATED WORK}

While the authors were unable to find any extensive classifications that differentiate the full range of human manipulation behaviors from one another, a number of related works should be mentioned.

There has been substantial earlier effort at classifying and categorizing human grasp types, without thorough treatment of how those relate to manipulation of objects being grasped. The first was by Schlesinger et al., which categorized grasps into six types: cylindrical, tip, hook, palmar, spherical, and lateral [2]. These grasps are primarily defined by the object that the hand interacts with. However, human grasps are often less dictated by size and shape of the object, but more by the tasks that need to be accomplished. In 1956, Napier suggested a scheme that would divide grasps into power and precision grasps [3]. Unfortunately not all the grasps fell cleanly into either of these two categories, with the lateral pinch in particular serving both power and precision functions.

In studying the grasps required for manufacturing tasks, Cutkosky provided a much more comprehensive and detailed organization of human grasps [4]. This taxonomy was acquired through a set of observational surveys on professional machinists and is essentially an integration of the previous work done by Schlesinger and Napier. The Cutkosky taxonomy tree is first divided into power and precision grasps from left to right, and by shape and function down the tree.

A small number of successive taxonomies, built primarily from the Cutkosky taxonomy, have been proposed (a comprehensive overview can be found in [5]). However, these 
include mostly minor variations and have not yet been extensively adopted by the research community. A preliminary investigation of the frequency of use of various grasp types in daily activities is presented in [6]. This study showed that a small subset of grasp types (6 for one subject and 9 for another) were used $80 \%$ of the time for the two subjects over the period examined.

Although we were unable to find any manipulation taxonomies that describe a full range of manipulation behavior, some related classifications should be discussed.

The two most closely related classifications are Elliott and Connolly's classification of intrinsic hand movements [7] and Exner's alternate classification [8]. Elliott and Connolly described three general classes of within-hand (intrinsic) manipulation movements: simple synergies, reciprocal synergies, and sequential patterns. In simple synergies, all digits involved move as one unit, such as while pinching or squeezing. In complex synergies, the fingers move together, but the thumb moves independently. Rolling an object between the thumb and the side of the index finger is an example of a complex synergy. Complex synergies are further subdivided by the principal direction of thumb motion. In sequential patterns, the digits move independently in a repeatable sequence. This system uses a hand coordinate system defined in terms of anatomical directions. One drawback of Elliott and Connolly's system is that it does not apply to all manipulation tasks - the authors note, for example, that flexor/extensor movements of individual digits are not included.

Exner's alternate classification has been used fairly extensively in clinical settings, but simplifications were made to ease clinical application that make it less suitable for research. He classified within-hand manipulation into five categories: palm-to-finger translation, finger-to-palm translation, shift, simple rotation, and complex rotation. The first three categories correspond in our proposed classification to translation motions along certain hand axes. The final two rotation categories would be described in our system as rotations about appropriate principal axes.

A much different but related classification is the taxonomy of haptic disassembly tasks [9] described by Bloomfield et al. in 2003. The proposed taxonomy classifies tasks according to task type and type of force or torque required. The force classification differentiates between tasks where the force is aligned with the motion, such as pressing a button, and those where the force is not aligned, such as sanding a surface. Torque is differentiated by whether the torque axis passes through the grip space, expressing the difference between turning a screwdriver and a steering wheel. However, the task type classification focuses on haptic simulation constraints, making it less appropriate in other contexts. For example, a significant force category is used because of the limited force capabilities of haptic devices, and a tool assisted category is used because the shaft of a haptic device often allows easier simulation of tasks in this category.
A.M Gentile's chapter in a 1987 book on movement science [10] proposes another task classification scheme. This taxonomy is based on environmental context and function of the action. It differentiates tasks using criteria such as whether the environment is static and whether there is environmental variation between trials. The taxonomy gives insight into the complexity of a task and can be used to evaluate a patient's movement capabilities. However, the only criterion describing the actual motion performed is whether a task is being performed in addition to basic body stability or body transport motions. Thus, our proposed classification would provide significant additional detail.

Various previous work has used the term manipulation primitives to describe components of a manipulation motion. The term is usually applied to the steps used in a specific algorithm or by a specific robot, but work by Morris and Haynes [11] describes a more general definition based on motion in 6 possible degrees of freedom between two rigid bodies. Morrow and Khosla [12] later improved on the notation used and described a general architecture for executing manipulation task primitives based on sensorimotor primitives defined based on a specific robot. These efforts focus on object motions and therefore differ substantially from the current hand-centric taxonomy.

Overall, although significant work has been done in classifying grasps and manipulation, no previous work has been found which attempts to classify the full range of human manipulation behavior. The previous classifications of withinhand manipulation found have some drawbacks, such as slightly limited scope, simplification for easy clinical use, or limited application outside of a specific context. Some of the classifications are also based on specific finger and thumb movements, which would prevent use of the classification to compare human and robotic hands.

\section{HUMAN MANIPULATION CLASSIFICATION}

Fig. 1 presents our manipulation taxonomy (with terms defined in section III.A. below). Note that in creating this classification, we take a hand-centric view of the problem, as opposed to an object-centric view. The taxonomy therefore focuses on what the hand is doing during execution of the manipulation task, rather than how the object being contacted is moving during the task. As a result of this classification, a given movement of an object can be done from multiple locations on the tree (e.g. a low-friction knob could be turned with a single finger as a non-prehensile task, or with multiple fingers as a prehensile task). Object-centric classifications might be made in a manner similar to [12], as described in section II above.

Note that this is also a motion-centric view of manipulation tasks, as opposed to a force-centric view (such as [9], as described above). However, it could be argued that they are essentially equivalent (i.e. force must be applied to produce motion and vice-versa). Tasks in which force is applied normal to the major direction of motion (e.g. writing on a 


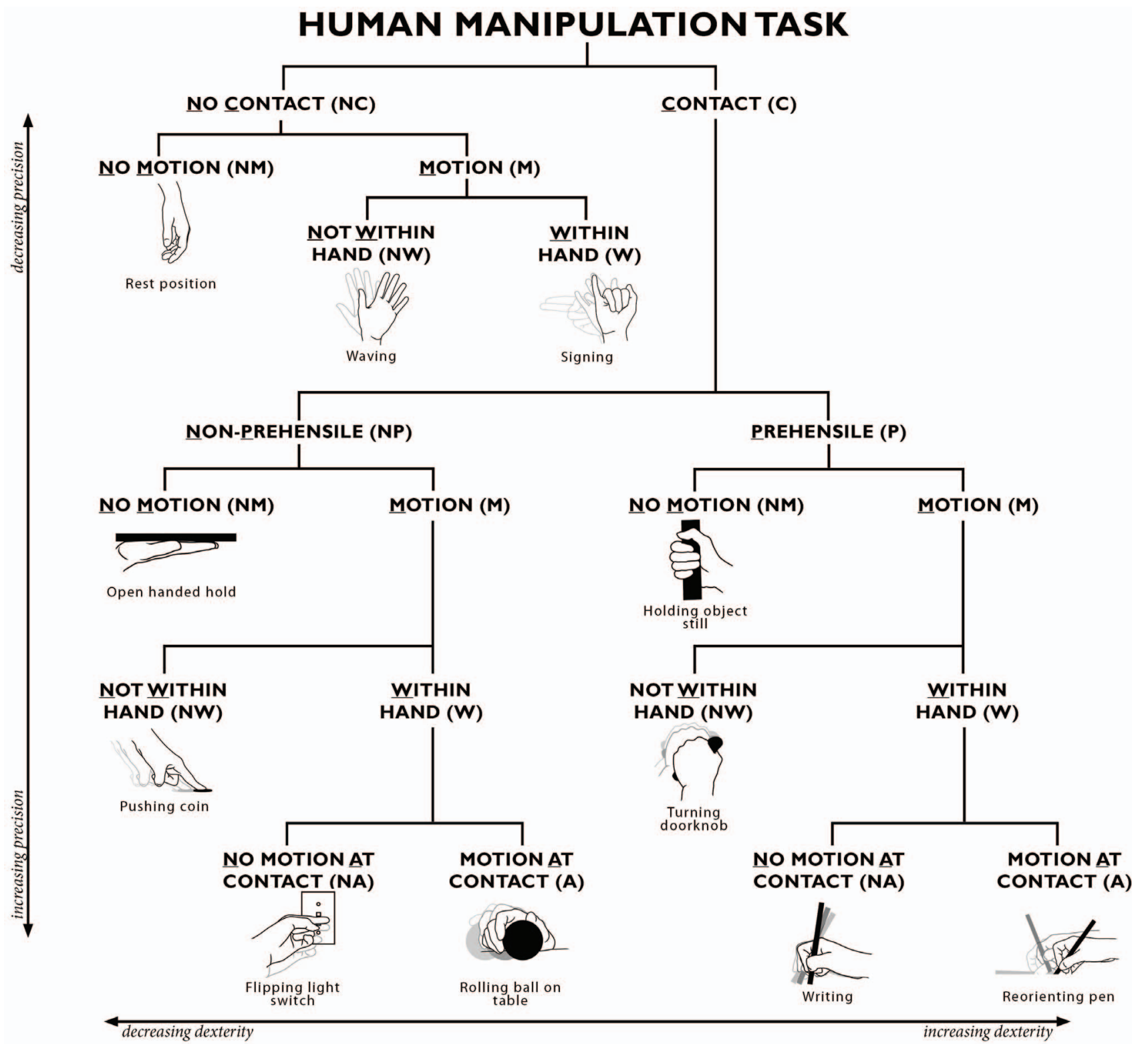

Fig. 1. Human manipulation taxonomy. Manipulation tasks are classified based on several criteria defined in section III.A. Representative examples are provided for each leaf of the tree.

board) would be considered two distinct simultaneous tasks, decomposed in a manner outlined in section III.D, below.

\section{A. Definition of Terms}

The following provides the definitions of the terms used in the taxonomy for each sub-classification. Since this taxonomy is "hand-centric," the majority of the terms describe the nature of the hand's interaction with external objects.

- Contact - The hand is touching an external object.

- Prehensile - Hand contact with an object is prehensile if it cannot be reasonably represented by a single contact point ("virtual finger" [4]). Equivalently, contact is prehensile if the contact forces from the hand alone can stabilize the object, regardless of external forces such as gravity.
- Motion - The hand is moving with respect to a body coordinate frame, such as the torso. A 'world frame' is not used in order to distinguish from cases where the whole body is moving, such as on an escalator.

- Within Hand - Motion occurs within the hand, and therefore parts of the hand, such as the fingers, are moving with respect to a frame fixed to the base of the hand. As a counter example, motion does not occur within the hand in cases where the hand is purely securing the object to the arm, such as power grasps. In these, the motion of the contacted object is primarily affected by the arm or rest of the body, but not by the hand. 
- Motion at Contact - The hand is significantly translating or rotating the object (or a combination of both) with respect to a frame affixed to the contact location(s) on the hand. Most manipulations involve some small contact changes due to skin deformation (such as writing with a pen), but these can be considered unintentional and not significant. Cases where the contact point stays the same, but the object is being pivoted, would be classified as 'motion at contact', since points on the object move relative to the fixed contact frame(s).

- Dexterity - Loosely defined as the complexity of the manipulation task, or the skill involved in performing a manipulation task.

- Precision - Loosely defined as the position accuracy able to be accomplished with the given configuration and category.

\section{B. Examples and Discussion of Categorization}

In this section, we discuss each branch of the taxonomy tree and provide examples of manipulation behaviors that fall within each, from left to right (less dexterous to more dexterous). Additionally, Table I lists a number of manipulation examples that fall into each category, exceeding those listed in Fig. 1.

- No Contact/No Motion - The hand is not moving and not contacting another object (e.g. in the rest position).

- No Contact/Motion/Not Within Hand - The hand is not contacting another object, is moving in space, but not reconfiguring (e.g. waving).

- No Contact/Motion/Within Hand - The hand is not contacting another object, but is itself reconfiguring (e.g. gesturing or signing).

- Non-Prehensile/No Motion - The hand is statically touching another object (e.g. leaning on a table or holding an object open-handed).

- Non-Prehensile/Motion/Not Within Hand - The hand is moving a contacted object, but is itself not reconfiguring and therefore acts as a passive link to the arm (e.g. pushing a large box or sliding a coin across a table).

- Non-Prehensile/Motion/Within Hand/No Motion at Contact - The hand is moving a contacted object and is itself reconfiguring, but without significant relative motion at the contact point(s) (e.g. typing on a keyboard, playing piano).

- Non-Prehensile/Motion/Within Hand/Motion at Contact - The hand is moving a contacted object, is itself reconfiguring, and significant relative motion at the contact point(s) is being produced (e.g. rolling a ball of dough on a table, turning a knob with a single finger).

- Prehensile/No Motion - The hand is statically grasping another object (e.g. holding a glass).

- Prehensile/Motion/Not Within Hand - The hand is moving a grasped object, but is itself not reconfiguring
TABLE I

COMMON MANIPULATION TASKS

\begin{tabular}{|c|c|}
\hline Category & Example Tasks \\
\hline \multirow{2}{*}{ No Contact/No Motion } & Hand in rest position \\
\hline & Blocking out sun \\
\hline $\begin{array}{l}\text { No Contact/Motion/ } \\
\text { Not Within Hand }\end{array}$ & Waving \\
\hline $\begin{array}{l}\text { No Contact/Motion/ } \\
\text { Within Hand }\end{array}$ & Signing \\
\hline \multirow{3}{*}{$\begin{array}{l}\text { Non-Prehensile/ } \\
\text { No Motion }\end{array}$} & Open handed hold \\
\hline & Leaning on table \\
\hline & Tactile exploration of surface* \\
\hline \multirow{2}{*}{$\begin{array}{l}\text { Non-Prehensile/Motion/ } \\
\text { Not Within Hand }\end{array}$} & Sliding coin across table \\
\hline & Pushing large box \\
\hline \multirow{3}{*}{$\begin{array}{l}\text { Non-Prehensile/Motion/ } \\
\text { Within Hand/ } \\
\text { No Motion at Contact }\end{array}$} & Flipping light switch \\
\hline & Pushing button \\
\hline & Pushing piano key \\
\hline \multirow{2}{*}{$\begin{array}{l}\text { Non-Prehensile/Motion/ } \\
\text { Within Hand/ } \\
\text { Motion at Contact }\end{array}$} & Rolling ball between hand and table \\
\hline & Non-prehensile knob/lid turning \\
\hline \multirow[b]{2}{*}{ Prehensile/No Motion } & Holding object still \\
\hline & $\begin{array}{l}\text { Doing a pullup (with motion at } \\
\text { contact)* }\end{array}$ \\
\hline \multirow{5}{*}{$\begin{array}{l}\text { Prehensile/Motion/ } \\
\text { Not Within Hand }\end{array}$} & Turning doorknob \\
\hline & Wiping surface \\
\hline & Pushing broom \\
\hline & Knife chopping \\
\hline & Power turn (e.g. loosening large lid) \\
\hline \multirow{7}{*}{$\begin{array}{l}\text { Prehensile/Motion/ } \\
\text { Within Hand/No } \\
\text { Motion at Contact }\end{array}$} & Writing \\
\hline & Precision lid turning \\
\hline & Inserting key \\
\hline & Precision lift \\
\hline & Cutting with $\mathrm{x}$-acto ${ }^{\circledR}$ knife \\
\hline & Using scissors \\
\hline & Squeezing sponge \\
\hline \multirow{3}{*}{$\begin{array}{c}\text { Prehensile/Motion/ } \\
\text { Within Hand/Motion at } \\
\text { Contact }\end{array}$} & Reorienting pen \\
\hline & Precision to power grasp transition \\
\hline & Regrasping \\
\hline
\end{tabular}

and therefore acts as a passive link to the arm (e.g. scrubbing with a sponge, turning a doorknob).

- Prehensile/Motion/Within Hand/No Motion at Contact - The hand is moving a grasped object and is itself reconfiguring, but without significant relative motion at the contact point(s) (e.g. writing with a pen, inserting a key into a lock, turning a small knob).

- Prehensile/Motion/Within Hand/Motion at Contact The hand is moving a grasped object, is itself reconfiguring, and significant relative motion at the contact point(s) is being produced (e.g. reorienting a pen into the writing position, transferring a screwdriver from a fingertip grasp to a power grasp).

The last two sections, comprising within hand prehensile manipulation, are described in more detail in section IV. 


\section{Further Sub-Categorization}

Using the existing categorization criteria for the taxonomy, certain further sub-categories might be added depending on the specific application of the taxonomy. For example, for each sub-categorization that includes motion of the object, more specific details of the nature of that motion can be added. These motions might be broken up by rotations or translations about some fixed frame, as is presented for dexterous within hand manipulation in section IV, or with regards to how many degrees of freedom in which the object can be actively manipulated in (similar to [12]). Alternatively, some type of classification related to the force, similar to [9], might be made.

\section{Classifying Complex Tasks}

The taxonomy above provides a structured way of classifying relatively simple tasks. More complex tasks have less obvious categorizations. There are three major types of complex manipulation tasks that require further discussion as to their categorization: time-separated sequences, simultaneous bi-manual tasks, and simultaneous within-hand tasks.

Time-separated motions, such as a long sequence of movements to accomplish an overall goal, should be classified as the sum of the discrete sub-components of the manipulation process. For instance, picking up a pen and writing with it might be decomposed into three sub-tasks: lifting the pen from the table (prehensile/motion/not within hand), rotating the pen into the writing position (prehensile/motion/within hand/motion at contact), and writing with it (prehensile/motion/within hand/no motion at contact).

Bi-manual tasks, where both hands are in use and required to perform the task, would be defined by the individual tasks being performed by each hand. Simultaneous use of the hands to perform independent tasks should not be considered 'bimanual'.

Tasks where the hand performs two or more simultaneous functions (e.g. pulling a hand drill trigger, thumb-typing on smartphone, using calipers, writing on a chalkboard, etc.) are some of the most dexterous tasks regularly performed. We propose that these types of tasks should be treated as the sum of the distinct sub-tasks being performed. For example, the task of pulling the trigger on a power drill could be categorized as a prehensile, no motion task (grasping and holding the drill handle) combined with a nonprehensile/motion/within hand task (index finger compressing the trigger). Thumb-typing on a cell phone would be similarly considered as the sum of a prehensile, no motion task (holding the phone with the palm and fingers) combined with a nonprehensile/motion/within hand task (thumb pressing the keys). Writing on a chalkboard, where a force is being applied to the board (to maintain contact) and the chalk is being moved along the surface of the board would be considered as the sum of two prehensile/motion/within hand tasks, as applying force to the board and moving the chalk both require actuation in each direction.

\section{DEXTEROUS, WiTHIN HAND MANIPULATION}

The term "dexterous manipulation" is used frequently in the robotics community, but no widely accepted definition exists. Perhaps the most common use, however, refers to manipulations that would be categorized as prehensile, within hand manipulation according to the taxonomy presented above. Indeed, there is great interest in the field to impart greater dexterity to robotic and prosthetic hands in the form of "within hand" manipulation capability. In this section we further discuss and sub-categorize this area.

Fig. 2 shows the dexterous manipulation taxonomy. We sub-categorize the movements according to the axis about which the movement is primarily concentrated, with respect to a coordinate frame affixed to the back of the hand. Each movement subcategory ("no motion at contact" and "motion at contact") is therefore expanded to three rotational and three translational movements with respect to this coordinate frame (plus some positional offset).

Due to constraints imposed by hand kinematics, it is unlikely that any movement would precisely align with the fixed coordinate frame axes. Instead, these are intended to be approximate. For movements that are significantly askew from these axes, a linear combination of cardinal movements might be used to describe the task.

Based on the constraints inherent with human hand kinematics, it is difficult to affect dexterous translational movements in the $\mathrm{x}$-direction or rotational movements about the $y$-axis. Indeed, there are few identifiable common dexterous manipulation tasks for those axes.

\section{CONCLUSIONS AND FUTURE WORK}

This paper provides a hand- and motion-centric categorization of human manipulation. Although previous work was found that classifies grasps or certain general aspects of manipulation, the proposed taxonomy provides a detailed categorization for human hand behavior. The taxonomy may be applied in various ways.

The proposed classification scheme might enable better understanding of human hand use. For example, the taxonomy emphasizes hand-centric differences between tasks that might appear similar if only object motion is considered. In some cases, a similar range of object motion might be accomplished through a "not within hand" or "within hand" strategy, with significant differences in the required hand dexterity. This might be used, for example, to help identify hand intensive tasks that a patient recovering from a hand injury should do carefully or sparingly. 


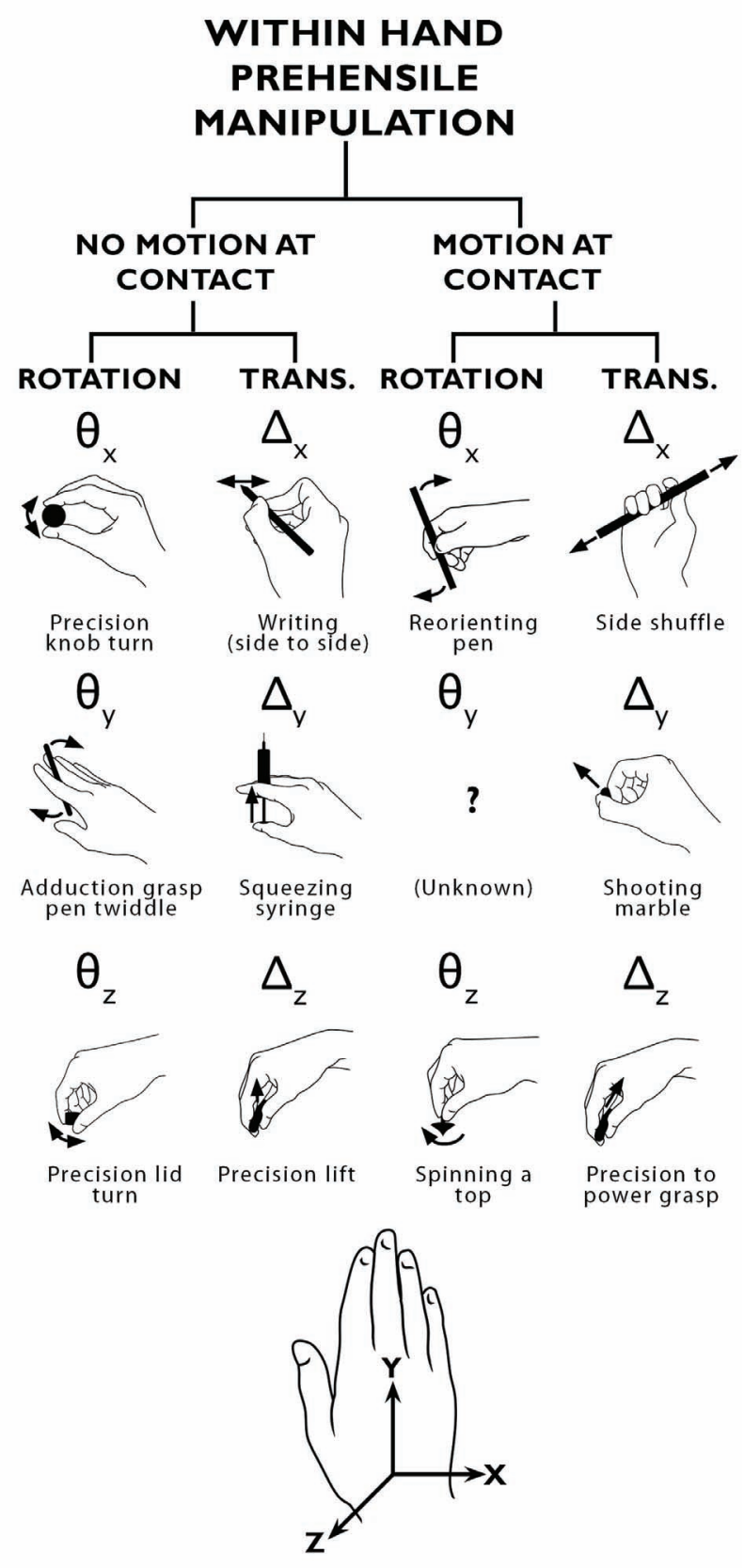

Fig. 2. Dexterous manipulation sub-categorization. Tasks are further classified by their principal axis of motion.

The taxonomy might also guide the creation of a set of standard manipulation tasks for each leaf of the tree. This kind of set should include the most frequent tasks that humans perform, and span a wide variety of practical hand motions. Although creating a complete set may be difficult or impossible, even an incomplete set might be a powerful tool for evaluating manipulation performance. This standard set could be used to evaluate a human patient's manipulation ability or chart their progress during use of rehabilitative devices, or to compare the dexterity of robot hands. In either case, performance on the standard task set could be used to assign an overall dexterity score to a hand, providing a structured basis for comparing hand performance.

\section{ACKNOWLEDGMENT}

This work was supported in part by DARPA grant W91CRB-10-C-0141 (ARM-H).

The authors would like to thank Lael Odhner, Raymond Ma, Josh Zheng, Leif Jentoft, and Sara de la Rosa for their helpful discussions related to the concepts presented in this paper, and to Kayla Matheus and Heidi Hong for helping to create the hand drawings used in the figures.

\section{REFERENCES}

[1] A. Faisal, D. Stout, J. Apel, and B. Bradley, "The Manipulative Complexity of Lower Paleolithic Stone Toolmaking," PLoS ONE, vol. 5(11), 2010.

[2] G. Schlesinger, "Der Mechanische Aufbau der Kunstlichen Glieder," in Ersatzglieder und Arbeitshilfen, M. Borchardt et al., Eds. Berlin, Germany: Springer, 1919.

[3] J. Napier, "The prehensile movements of the human hand," J. Bone Joint Surg., vol. 38B, no. 4, pp. 902-913, Nov. 1956.

[4] M.R. Cutkosky, "On Grasp Choice, Grasp Models, and the Design of Hands for Manufacturing Tasks," IEEE Trans. Robot. Automat., 5(3), (1989) 269-279.

[5] T. Feix, Otto Bock. "Human Grasping Database." Internet: http://web.student.tuwien.ac.at/ e0227312/, [Sep. 10, 2010].

[6] J. Z. Zheng, S. De La Rosa, and A. M. Dollar An Investigation of Grasp Type and Frequency in Daily Household and Machine Shop Tasks, Proceedings of the 2011 IEEE International Conference on Robotics and Automation (ICRA), Shanghai, China, May 9-13, 2011.

[7] J.M. Elliott and K. Connolly, "A classification of manipulative hand movements," Developmental Medicine \& Child Neurology, vol. 26, p. 283-296, 1984.

[8] C.E. Exner, "In-hand manipulation skills. In: American Occupational Therapy Association (Ed.). Development of hand skills in the child (pp. 35-45). Rockville, MD: American Occupational Therapy Association, 1992.

[9] A. Bloomfield, Y. Deng, J. Wampler, P. Rondot, D. Harth, M. McManus, and N. Badler, "A taxonomy and comparison of haptic actions for disassembly tasks," Virtual Reality, 2003. Proceedings. IEEE, IEEE, 2003, p. 225-231.

[10] J.H. Carr, R.B. Shepherd, J. Gordon, A.M. Gentile, and J.M. Held, Movement Science: Foundations for physical therapy in rehabilitation, Rockville,: Aspen Publishers, Inc., 1987.Sdf

[11] G. Morris and L. Haynes, "Robotic assembly by constraints," Proceedings. 1987 IEEE International Conference on Robotics and Automation, 1987, pp. 1507-1515.

[12] J.D. Morrow and P.K. Khosla, "Manipulation task primitives for composing robot skills," Proceedings of International Conference on Robotics and Automation, 1997, pp. 3354-3359. 